\title{
Autobiographical Memory in a Fire-Walking Ritual
}

\author{
Dimitris Xygalatas ${ }^{a, b, c, d, *}$, Uffe Schjoedt ${ }^{a, b, c}$, Joseph Bulbulia ${ }^{c, d, e}$ \\ Ivana Konvalinka ${ }^{\text {b,f }}$, Else-Marie Jegindø ${ }^{a, c, f}$, Paul Reddish $^{\mathrm{d}, \mathrm{e}}$, \\ Armin W. Geertz ${ }^{a, b, c, f}$ and Andreas Roepstoff $a, b, f$
}

a Department of Culture and Society, Aarhus University, 8000 Aarhus, Denmark

b Interacting Minds Centre (IMC3), Aarhus University, 8000 Aarhus, Denmark

c Religion, Cognition and Culture Research Unit (RCC), Aarhus University, 8000 Aarhus, Denmark

d LEVYNA Laboratory for the Experimental Research of Religion, Masaryk University, Czech Republic

e Religious Studies Department, Victoria University, Wellington, New Zealand

${ }^{f}$ Centre for Functionally Integrative Neuroscience (CFIN),

Aarhus University Hospital, 8000 Aarhus, Denmark

* Corresponding author, e-mail: xygalatas@mac.com

\begin{abstract}
Anthropological theories have discussed the effects of participation in high-arousal rituals in the formation of autobiographical memory; however, precise measurements for such effects are lacking. In this study, we examined episodic recall among participants in a highly arousing firewalking ritual. To assess arousal, we used heart rate measurements. To assess the dynamics of episodic memories, we obtained reports immediately after the event and two months later. We evaluated memory accuracy from video footage. Immediately after the event, participants' reports revealed limited recall, low confidence and high accuracy. Two months later we found more inaccurate memories and higher confidence. Whereas cognitive theories of ritual have predicted flashbulb memories for highly arousing rituals, we found that memories were strongly suppressed immediately after the event and only later evolved confidence and detail. Physiological measurements revealed a spectacular discrepancy between actual heart rates and self-reported arousal. This dissociation between subjective reports and objective measurements of arousal is consistent with a cognitive resource depletion model. We argue that expressive suppression may provide a link between individual memories and cultural understandings of high-arousal rituals.
\end{abstract}

\section{Keywords}

Arousal, episodic memory, expressive suppression, fire-walking, flashbulb, ritual 


\section{Introduction}

The relationship between arousal and memory has in recent decades become a prominent theme in ritual theory. The question of how highly arousing rituals affect memory, however, has not been assessed quantitatively. Cognitive theories of ritual have stressed the role of memory in the transmission of ritual traditions (Whitehouse, 1992; 2004; McCauley and Lawson, 2002), particularly focusing on the role of flashbulb memories. In particular, Harvey Whitehouse (2004: 71) has argued that "rarely performed and highly arousing rituals invariably trigger vivid and enduring episodic memories... These memories can be so vivid and detailed that they can take the form of ... flashbulb memories. It is almost as if a camera has gone off in one's head, illuminating the scene, and preserving it forever in memory". This hypothesis is based on findings from memory research that some highly stressful and emotionally arousing experiences produce episodic memories in which events are recalled with extraordinary vividness (Brown and Kulik, 1977). A flashbulb memory hypothesis for ritual, then, predicts quasi-perceptual memories for highly arousing ritual events.

There are, however, several plausible reasons for doubting the adequacy of the flashbulb hypothesis for ritual memories. This "photographic" model of episodic memory is a highly ambiguous one in psychological literature (see McCauley and Lawson, 2002). So-called "flashbulb memories" have mainly been studied in the context of unexpected, highly unusual and often affectively negative events, which typically lack prescribed cultural interpretations and schemas. Rituals, by contrast, are generally (with few exceptions) anticipated, predictable, and highly scripted events. While certain details of the ritual experience will be surprising, many others are foreseen or rehearsed. Interpretations are typically culturally inflected, which may affect the elaboration of episodic memories. Indeed, it is a general finding in memory research that cultural expectations affect both interpretations and recollections (Bartlett, 1932; Berntsen and Rubin, 2004; Collins et al., 2007. For a discussion, see Ward, 1984: 324-326). Notably, ritual events also often (though not always) impose rules on the expression of emotions, and some of these rules may directly work against the encoding of vivid episodic memories. For example, expressive suppression of strong emotions has been demonstrated to impair memory retention in response to emotionally arousing stimuli (Richards and Gross, 1999, 2000; Richards, 2004).

These findings point to two divergent hypotheses for the mechanisms involved in the production of the rich autobiographical memories of highly arousing rituals so often reported by anthropologists (Xygalatas, 2012). A flashbulb hypothesis suggests that emotionally arousing ordeals will imprint vivid 
and detailed episodic memories (Whitehouse, 2002, 2004). On the other hand, a suppression hypothesis would predict that highly arousing rituals result in limited memory recall, which later evolves with culturally mediated inflections.

To our knowledge, no previous study has accurately measured arousal levels and memory formation in a real-life ritual. Whereas previous field studies have been limited to observational work by ethnographers, reproducing high arousal rituals in the laboratory is problematic. Not only do such laboratory studies of sacred rituals often face ethical issues, but they also lack ecological realism because the potential effects of cultural expectations, emotion regulation and religious meanings attributed to the ritual are unlikely to reproduce in an artificial setting. We therefore decided to incorporate relevant laboratory methods and equipment into traditional fieldwork to obtain measurements in a natural context. Our findings are relevant not only to anthropological theories of ritual memories, but also to a wider memory literature. At the same time, such findings answer a call from certain quarters of memory research for greater ecological realism.

\section{Methods}

\section{Setting}

The study was conducted in San Pedro Manrique, a small Spanish village located approximately $200 \mathrm{~km}$ Northeast of Madrid. On the $23 \mathrm{rd}$ of June every year, the local inhabitants conduct a fire-walking ritual as part of the festival of San Juan, which incorporates both religious and secular themes (Iñigues y Ortiz, 1924; Caro Baroja, 1950; Cortés, 1969; Ruiz Vega, 1985; San Baldomero Ucar, 1998). The venue for this event is an amphitheatre that has been built specifically to host the ritual, with the capacity to accommodate up to 3000 visitors, roughly five times the village population. The ritual starts with a 15-minute procession, during which participants briskly climb a steep hill leading to the venue. Upon entering, fire-walkers are greeted by a searing hot carpet of glowing red coals, about 7 metres long, one metre wide, and about $20 \mathrm{~cm}$ deep. Using a pyrometer, we measured the surface temperature of the coal bed at $677^{\circ} \mathrm{C}$. The fire-walkers form a circle and dance around the fire. After circumambulating the fire for several minutes, they assemble, each awaiting a turn to walk the fire. Walkers are beckoned to their trial by the sound of a trumpet burst. The fire-walk is conducted bare-footed (Fig. 1). While crossing the fire, most walkers carry one of their beloved persons on their backs. Once the walker and passenger traverse the coal bed, their family and closest friends rush over to hug them and congratulate them. 


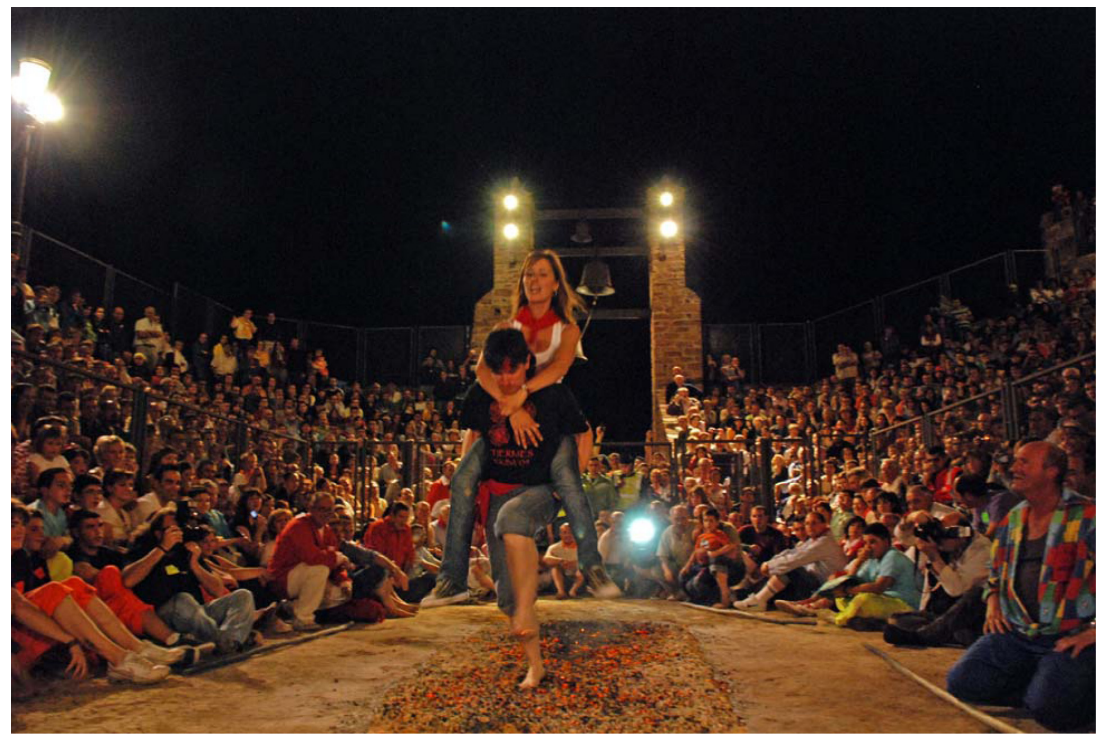

Figure 1. The fire-walking ritual. This figure is published in colour in the online edition of this journal, which can be accessed via http://booksandjournals. brillonline.com/content/15685373.

While fire-walking is not attached to any Christian tradition, some San Pedrans believe that the Virgin Mary intercedes to protect the walkers. This belief provides an explicitly religious interpretation for the event. However, other participants do not attach any supernatural meaning to the ritual. Yet all participants reported fire-walking to be fundamental to their individual and collective identity as San Pedrans, and all judged the ritual to be of the highest personal and collective salience. Specifically, when we asked participants to rate the importance of this ritual to them on a scale from 1 to 10 , where 10 is the highest possible importance, they expressed a mean-rated importance of 8.14 $(n=14, \mathrm{SD}=1.10)$. Spontaneous comments from the structured and unstructured interviews also supported these high valuations:

a) "San Pedro would not be San Pedro without the fire-walk."

b) "This is our tradition; it's our identity; it's part of who we are."

c) "During the whole year I look forward to this day; and when this day is gone, then I await for it to happen again the next year."

d) "It's a feeling of pride; it's very important. Me, every single time, after fire-walking, I burst into tears." 
e) “- How important is fire-walking to you? - Very, very much. - How important would you rate it on this scale, from ... - (interrupting) The maximum. - How important would you rate fire-walking on a scale from 1 to 10 ? - Extremely important. Out of $10 \ldots$ if you marked 20 on your scale, you'd be right!"

\section{Participants}

On the day of the ritual, data were obtained from 12 (of a total of 28) firewalkers (11 male and 1 female, mean age $=32.5$ years, range $19-46, \mathrm{SD}=10.90$ ). Note that the majority of participants were male and wherever there are any variations in sample size throughout this paper, this is because some participants were unable or chose not to participate in certain components of the study (e.g., not to wear a heart rate monitor). Written approval for the study was obtained by the Town Council and informed consent was granted by all participants.

\section{Arousal Measurements}

We operationalised arousal in terms of autonomic activity (heart rate). Heart rate data were obtained from Polar Team transmitter belts worn around the chest. Recordings were taken for a continuous period of $2-3$ hours, which included 30 minutes well before the event (baseline), the procession, and the entire fire-walking ritual (27 minutes in length). (for more on these measurements, see Konvalinka et al. 2011) After the ritual, participants also provided self-reported ratings of arousal for the event.

\section{Memory Measurements}

The ritual was recorded using five high-definition video cameras synchronized to the heart rate monitors. Video footage enabled us to test the accuracy of participant memory reports. A combination of free recall and structured memory reports were obtained within two days following the fire-walk, and again after a two-month delay (henceforth $t_{1}$ and $t_{2}$, respectively). A free recall task was used to evaluate unique memories of the event. We presented this task first, in order to avoid invoking pattern completion (McClelland, 1997) and similar cueing effects. Participants were asked to recall as many details as they could from the fire-walking event, with the clarification that they should mention memories of their specific performance that year.

Transcripts of participant reports were evaluated by two independent raters, unaware of the research hypotheses. Coders were instructed to count the 
number of factual details reported by each participant in the free recall memory conditions at $t_{1}$ and $t_{2}$ (inter-rater reliability: intra-class correlation $=0.921$ ). Disagreements were subsequently discussed between the raters until consensus was reached. A factual detail was defined as an observable, verifiable event, specific to a participant's fire-walk. A participant's fire-walk was defined as the period between when the participant stood up and approached the fire to the time when fire-walker sat down after completing their trial and embracing family and friends. Remembering the person the fire-walker carried (which carriers often know months in advance), or mental thoughts or emotions detached from the verifiable qualities of the event were not counted as factual details. Coders independently rated the emotional content of the free recall reports for both the recollection of emotion and the valence of the emotion. The presence of emotion was coded either by the expression of a specific emotion (e.g., "I was nervous") or by the expression of a general emotion (e.g., "it was very overwhelming") (inter-rater reliability: Kappa=0.898). The valence of emotion was coded as positive, negative, neutral/undetermined, or no emotion (interrater reliability: Kappa $=0.648$ ).

After the free-recall task, two structured questions targeted recall for verifiable spatial, temporal and social distributions and sequences of the event. Participants were asked to recollect the identities of those who were seated nearest to them before their fire-walk. The number of facts reported in such accounts was used as a measure for structured recall. The same coders from the free recall task also coded the structured recall task. Besides counting facts, coders were asked to rate confidence for each report as either "confident" or "not confident" (inter-rater reliability: Kappa=0.882). Confidence was determined by the presence of phrases such as "I guess" and "I don't remember" related to factual assertions. A rater unfamiliar with the research hypothesis determined accuracy for both unstructured and structured memory reports by comparing statements at $t_{1}$ and $t_{2}$ with video footage.

\section{Results}

\section{Arousal}

Heart-rate measurements revealed that fire-walking activated an extremely strong physiological response in all performers. Absolute maximum heart rates during the walk varied between 165 and 193 beats per minute $(M=178.90$, $\mathrm{SD}=8.81$ ). For every participant, peak heart rate invariably occurred during their walk and exceeded the heart rate levels from jogging up a steep hill and dancing before the event. One-way repeated measures ANOVA revealed 


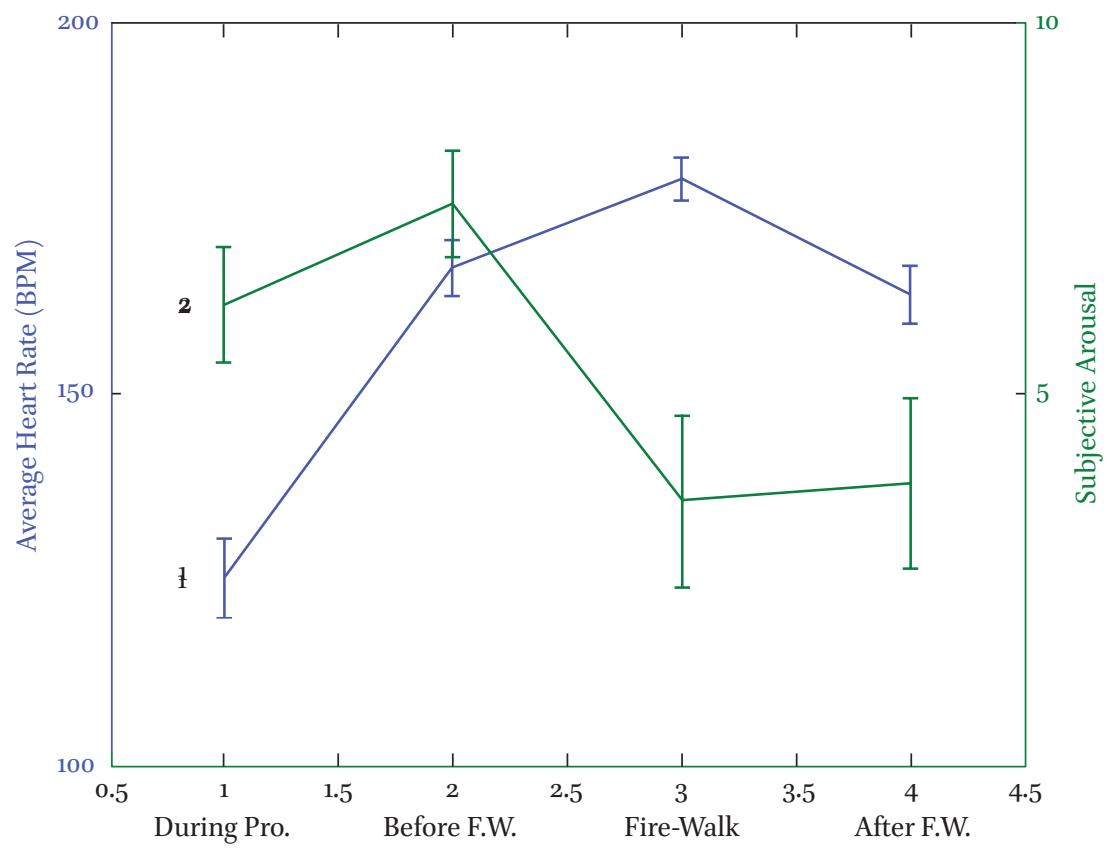

Figure 2. Comparison between heart rate measurements (1, blue line) and selfreported arousal (2, green line). The first time point includes the procession towards the venue, partly climbing a steep hill at a fast pace; the second time point includes dancing inside the venue and waiting for the fire-walk; the third time point refers to the time of the individual fire-walks; and the fourth time point is approximately 10 minutes after each fire-walk. Note that results have been normalized, as fire-walking was performed serially by participants (one by one), while for the rest of the ceremony participants acted in parallel. Thus, the continuous lines are provided for visual purposes only, as they do not represent the actual fluctuations between time points, which represent mean numbers. This figure is published in colour in the online edition of this journal, which can be accessed via http://booksandjournals.brillonline.com/ content/15685373.

a significant difference in arousal during the four time intervals (procession, before the fire-walk, during the fire-walk, and after the fire-walk). Tukey's HSD (Honestly Significant Difference) tests revealed higher arousal during the firewalk than during any other time interval. For $66 \%$ of participants, peak heart rate during the fire-walk exceeded the conventional safe maximal heart rate $\left(\mathrm{HR}_{\max }\right)$ (Londeree and Moeschberger, 1982; Robergs Landwehr, 2002). 
However, this biomarker of arousal strongly contrasted with subjective ratings obtained after the ritual, which suggested a very different pattern. On a scale of 1 to 10 , where 10 is the maximum possible arousal, mean arousal reports for the procession prior to the fire-walk (first interval) was 6.20 ( $\mathrm{SD}=2.44$ ); mean reported arousal for the time between entering the venue and performing the fire-walk (second interval) was $7.55(\mathrm{SD}=2.25)$; remarkably, mean reported arousal for the duration of the fire-walk (third interval) was 3.55 ( $\mathrm{SD}=3.47$ ); finally, reported arousal for the period immediately after the walk (fourth interval) was $3.80(\mathrm{SD}=3.65)$ (Fig. 2). There was no correlation between subjective reports of arousal and actual arousal. In the interviews, $92 \%$ of participants explicitly predicted that their heart-rate would prove to be lower during the fire-walk even compared to their relaxed baseline measurements. In fact, several participants were willing to bet money that their heart rates during the ritual would be at minimal levels, and indeed challenged the experimenters to take those bets (which we politely refused).

\section{Memory}

Unstructured Recall Reports. Unstructured memory reports were based on the free recall details that participants reported concerning their individual firewalks. Factual memories obtained from the unstructured $t_{1}$ accounts were surprisingly scarce. A total of 5 factual details across all participants were recalled at $t_{1}$, while 7 out of 10 participants were unable to recall any specific details. Indeed, 4 participants explicitly described themselves as being "disconnected" or "blacked out" during their walk. Details of $t_{2}$ unstructured accounts were also scarce. A total of 8 details were recalled, with 5 participants unable to recall any details.

These results suggest that fire-walkers had difficulty freely recalling factual details and dynamics for their fire-walk. However, reports of affective recall were common at $t_{1}$. 8 out of 10 participants spontaneously recalled strong emotions. Such emotional memories appeared to decline at $t_{2}$, where only 4 participants provided similar statements. A McNemar test, however, found that this difference was not significant $\left.\chi^{2}(1, N=10)=2.35, p=0.13\right)$. Overall, $t_{2}$ reports seemed to be qualitatively different from $t_{1}$ reports, and tended to focus more on factual memories and less on affective memories, as in the example below:

$t_{1}$ : I remember feeling proud to be a San Pedran and to have the privilege to cross the fire. And that I was very content, I was thinking that I was not going to get burnt. I was thinking, "it's just me". I knew there were hundreds of people around me, but I felt that I was all alone. And then I don't remember anything. 
$T_{2}$ : I remember being impatient. I remember that Monica was the last person to walk before it was my turn. When my time came, I stood up, I hugged my father and I crossed the fire. I remember that my father, when he was on my back, he waived at the crowd. I crossed, I think I took 6 steps inside the fire. I felt being all alone, all the people disappeared, as if there was no one on my back nor around me. And then I was on the other side. Then I hugged my father hard. There were people around me... and... I don't remember anything else...

Structured Recall Reports. Structured questions assessed specific spatial and temporal memories for the period immediately before and after the fire-walk. Memories in the structured reports were also limited, with a mean number of details at $t_{1}=3.50(\mathrm{SD}=2.88)$ and at $t_{2}=5.20(\mathrm{SD}=2.86)$. Despite an increase in absolute numbers of reports, a paired sample t-test found that this increase was not significant.

To determine memory accuracy, we compared details of the reports to video footage of the event. While a greater number of facts were reported at $t_{2}$, accuracy declined. At $t_{1}, 66 \%$ of the facts given were accurate, while at $t_{2}$ only $40 \%$ were accurate. A paired samples $t$-test revealed that the mean number of accurate reports at $t_{1}(M=2.30, \mathrm{SD}=2.00)$ was not significantly higher than at $t_{2}$ $(M=2.1, \mathrm{SD}=2.33),(t(9)=0.29, p=0.78, d=0.09, r=0.05)$. However, the mean number of inaccurate reports did significantly increase from $t_{1}(M=1.20, \mathrm{SD}=2.20)$ to $t_{2}(M=3.10, \mathrm{SD}=2.47),(t(9)=2.69, p=0.03, d=0.81, r=0.38)$ (Fig. 3$)$. Although our

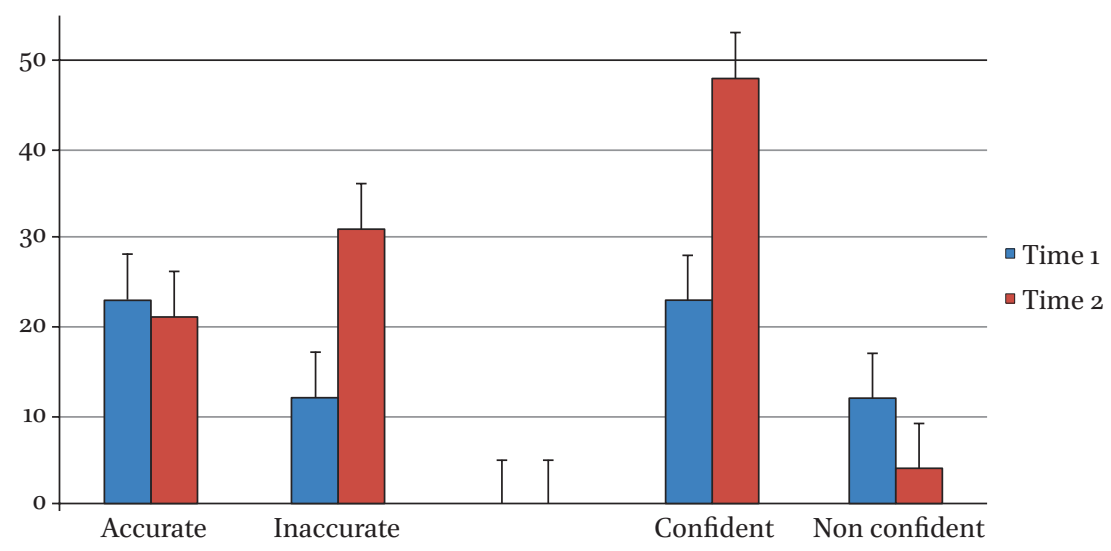

Figure 3. Number of facts recalled from structured recall reports at $t_{1}$ and $t_{2}$ classified as to the accuracy and confidence with which they were stated. This figure is published in colour in the online edition of this journal, which can be accessed via http://booksandjournals.brillonline.com/content/15685373. 
sample was small, this increase in the number of errors was evenly distributed and seemed to reflect a general trend which was not simply the result of one or two persons' inaccurate recall. This trend indicates that while there was loss of some factual details and gain of others, the overall trend was toward erroneous memories.

Somewhat contrary to expectations, confidence over the two-month interval also increased. At $t_{1}, 66 \%$ of the facts given were rated as confident, while at $t_{2}, 92 \%$ were rated as confident. Paired samples $t$-tests revealed that the increase in the mean number of confident reports from $t_{1}(M=2.30, \mathrm{SD}=2.79)$ to $t_{2}(M=4.80, \mathrm{SD}=3.19)$, was highly significant $(t(9)=3.16, p=0.01, d=0.83, r=0.38)$. On the other hand, there was no significant difference in the mean number of unconfident reports at $t_{1}(M=1.20, \mathrm{SD}=1.62)$ to $t_{2}(M=0.40, \mathrm{SD}=0.97),(t(9)=1.27$, $p=0.24, d=0.60, r=0.29)$. Thus, participants' memory of their trials by fire evolved over time to become more assured but also more prone to error.

\section{Discussion}

Field studies like the one presented here are always a challenging endeavour, as researchers typically cannot introduce or manipulate variables. The number of participants available was small, limiting the inferential power of our data. In addition, it was not possible to control for potentially confounding variables such as age, gender, health, lifestyle, alcohol intake, etc. The lack of control that the field imposes does not allow to isolate key variables or determine causal relations between aspects of the ritual and participants' memory with any kind of certainty. These restrictions reflect constraints intrinsic to field experimentation and emphasize the need for further investigation of our findings in more controlled environments. In spite of such limitations, however, anthropological experiments are critical to test whether theoretical insights and findings from controlled studies match actual dynamics in the field. Furthermore, such methods offer additional qualitative data which allow for triangulation and added inferential potential.

Our study is the first to quantify arousal and memory accuracy from a reallife event, not only in the literature on ritual but in psychology more general, answering calls to investigate episodic memory and emotion regulation in its natural social and ecological settings (Brewer, 2000; Crano and Brewer, 2002; Rimé, 2007; Gross, 2007; Butler and Gross, 2009). This is particularly important in the context of rituals, which occur in very specific social and cultural settings that may exhibit designs that offload cognitive tasks to the environments of interaction (Clarke and Byrne, 1993; Bulbulia, 2008; Vogeley and 
Roepstorff, 2009). Although we are convinced that field studies are necessary to test the external validity of laboratory findings, we also believe that new evidence and hypotheses derived from such field studies will have to be tested for internal validity in more controlled settings. We see the two approaches as complementary.

Two innovations are of methodological interest. First, we assessed recall accuracy using video recordings. This enabled us to precisely assess memory accuracy. Second, we investigated the relationship between arousal and memory using precise physiological measures. Our methodology allowed us to avoid reliance on self-reports, which would be unsatisfactory for our purposes, especially given our demonstration of inaccuracy both in memory and in perceived arousal. We consider the latter to be a crucial finding in itself, one which demonstrates the need for objective measures rather than the exclusive reliance on self-reports.

Our findings show that initial episodic recall for the ritual was poor and that participants tended to focus on affective qualities of the ritual. After two months, a different pattern was observed. Although more factual details were reported, errors significantly increased. Confidence, too, significantly increased. These findings do not support the hypothesis that participation in high arousal rituals will necessarily result in detailed and vivid flashbulb memories (Whitehouse, 1992, 2004). However, certain predictions of the flashbulb hypothesis do find support: The fire-walk was recollected in emotional terms; structured memories eventually did form; confidence eventually increased. On the other hand, few factual memories were recounted immediately after the event; low confidence for $t_{1}$ reports suggests quasi-perceptual details were initially lacking; and memories that were elaborated at $t_{2}$ were prone to error.

One possible interpretation of the participants' poor memory might be related to some form of stress-induced amnesia. Studies on traumatic experiences show that stressful events may sometimes lead to memory repression (Loftus and Kaufman, 1992; Bowman, 1996; Brown et al., 1998; Joseph, 1999; Bourget and Whitehurst, 2007; Kikuchi et al., 2010), and it has been proposed that such disruptions form part of an adaptive memory-repression response system designed to cope with traumatic memories by keeping them out of awareness (Freyd, 1994). This interpretation, however, does not correspond with the participants' claim that they felt calm during the fire-walk or with the qualitative interviews, where they describe the event as inherently positive. Furthermore, our video recordings showed that the participants consistently expressed great joy and enthusiasm after the fire-walk.

Another possible interpretation, which we find more plausible in light of memory research (see Berntsen and Rubin, 2007), is that the cultural framing 
of the ritual may have had important effects on how participants experienced and formed memories of the event. Our ethnographic work and qualitative interviews suggest that there was strong social motivation among practitioners to appear calm during their ordeal. The ceremony has a heroic character, and bravery is a pervasive theme. Participants often use expressions like "defeating" the fire, while various myths connect this custom to a triumphant military victory over the Arab conquerors in the Middle Ages (Cortés, 1969). Within this framework, expressions of fear or pain are signs of weakness or lack of divine protection. When performers take their place behind the fire, the thing they fear the most is presumably not the fire itself, but losing face in front of 3000 spectators, among whom most of their relatives, acquaintances, friends and potential mates. It is only when their ordeal is over that fire-walkers express strong (positive) emotional reactions. This desire to appear calm in a state of extreme physiological arousal may have important effects on memory. Research shows that efforts to suppress expressions of arousal may impair memory retention. Participants who experience the rush of arousal but attempt to conceal their feelings show impaired performance for cognitive tasks (Carver and Scheier, 1981). In particular, studies have demonstrated that emotional arousal can impede perceptual memory (Richards and Gross, 1999, 2000; Richards, 2004) and increase memories for emotional states (Richards et al., 2003). One explanation that has been proposed for this effect is that expressive suppression and memory retention compete for cognitive resources, a notion that corresponds with a general resource depletion model of executive function in which attention, working memory, and voluntary thought and behaviour utilise the same frontal regions in the human brain (Yerkes and Dodson, 1908; Ellis and Ashbrook, 1989; Christianson, 1992; Wegner et al., 1993; Engle et al., 1995; Kirchbaum et al., 1996; Baumeister et al., 1998; Macrae et al., 1998; Schjoedt et al., 2011, 2013). This effect may be especially relevant in highly arousing social events where cultural norms impose strong expressive suppression on participants. We propose that an attempt to conform to social expectations of emotion regulation may have contributed to participants' poor memory of the fire-walk.

Importantly, we observed an increase in participants' inaccurately reported memories two months after the ritual, which suggests that they continued to construct memories of the event in the weeks following the ritual. Our video recordings revealed that these memories were not in accordance with the actual events that took place, which indicates that these were based on cognitive elaboration rather than on retrieval of perceptual memories. We cannot determine whether these new constructions have been socially negotiated or if they simply express learned schemas on what most likely happened during 
the ritual (Berntsen and Rubin, 2004). However, our qualitative analysis suggests that the direction was towards more convergent patterns. This is to be expected, since the fire-walking ritual is typically the main topic of discussion in the village for several days and perhaps weeks following its performance, while people often watch videos and read reports of the event in the local Press. Finally, participants' higher confidence in their memories two months after the ritual further suggests that these new constructed memories had been consolidated in the practitioners' representation of the event. This is congruent with psychological literature pointing to a dissociation between confidence in the accuracy of flashbulb memories and the actual consistency of those memories (Talarico and Rubin, 2003).

Based on these findings, we propose that strong expressive suppression in rituals may have left participants' arousal-induced need for memory construction to be satisfied by schema-based or socially negotiated constructions rather than by idiosyncratic flashbulb memories. Since expressive suppression and detailed event schemas are characteristic to many rituals around the world, similar effects may be widespread. Furthermore, emotion regulation need not be the only mechanism in rituals to prevent practitioners from forming strong individual memories. Demanding behavioural procedures and exhausting verbal performances are commonly employed in rituals (Bloch, 1986; Rappaport, 1999; Liénard and Boyer, 2006; Boyer and Liénard, 2007). Such behaviours may have similar depleting effects on the executive system and thus function to impair individual memory and facilitate a collective mnemonic reconstruction of the event (Schjoedt et al., 2013).

We do not claim that these findings apply to all or even most high-arousal rituals. For example, where the cultural framing does not impose strong expressive suppression or employ other strategies to deplete cognitive resources of the executive system, rituals will generate similar memory effects to those of unexpected, unusual, and highly arousing events. However, our study reveals a plausible, hitherto unexplored alternative which might have important implications for our understanding of memory formation for highly structured emotional social events such as public rituals, allowing for memories to be elaborated over time even when initial recall for the event is inhibited.

\section{Conclusion}

Based on our findings, we propose that high arousal rituals may on the one hand promote arousal-induced memory construction but at the same time may also use expressive suppression to prevent individuals from forming strong 
perceptual memories. This mechanism leaves the arousal-induced memory formation empty for cultural schemas and socially negotiated constructions to fill the gap. We suggest that this mechanism may facilitate the construction of a canonical memory of collective rituals that matches the norms and beliefs of a given culture. Future studies will determine how common these observed effects are in high-arousal rituals across cultures and to what extent rituals may use other mechanisms that have similar effects on the participants' memory construction.

\section{Acknowledgements}

Support for this study was provided by the MINDLab UNIK initiative, the Centre for Functionally Integrative Neuroscience (CFIN), the Religion, Cognition and Culture Research Unit (RCC), and the Velux core research group Technologies of the Mind at Aarhus University; the Aarhus University Research Foundation; the Faculty of Humanities and Social Sciences at Victoria University, New Zealand; and by Polar Electro Inc. We thank Dorte Berntsen, Chris Frith and Uta Frith for their feedback, and Adriana Alcina Gomez for field assistance.

\section{References}

Bartlett, F. (1932). Remembering. A study in experimental and social psychology. Cambridge University Press, Cambridge.

Baumeister, R. F., Bratslavsky, E., Muraven, M. and Tice, D. M. (1998). Ego depletion: Is the self a limited resource? Journal of Personality and Social Psychology 74, 1252-1265.

Berntsen, D. and Rubin, D. C. (2004). Cultural life scripts structure recall from autobiographical memory. Memory and Cognition 32, 427-442.

- (2007). When a trauma becomes a key to identity: Enhanced integration of trauma memories predicts posttraumatic stress disorder symptoms. Applied Cognitive Psychology 21, 417-431.

Bloch, Maurice (1986). From blessing to violence: History and ideology in the circumcision ritual of the Merina of Madagascar. Cambridge University Press, Cambridge.

Bourget, D. and Whitehurst, L. (2007), Amnesia and crime. Journal of the American Academy of Psychiatry and the Law 35, 469-480.

Bowman, E. S. (1996). Delayed memories of child abuse. Dissociation: Progress in the Dissociative Disorders 9, 221-243.

Boyer, P. and Liénard, P. (2007). Why ritualized behavior? Precaution systems and action parsing in developmental, pathological and cultural rituals. Behavioral and Brain Sciences 29, 595-613.

Brewer, M. (200o). Research design and issues of validity. In Reis, H. and Judd, C. (Eds), Handbook of research methods in social and personality psychology, pp. 3-16. Cambridge University Press, Cambridge.

Brown, D. P., Scheflin, A. W. and Hammond, D. C. (1998), Memory, trauma treatment and the law. Norton, New York, NY.

Brown, R. and Kulik, J. (1977). Flashbulb memories. Cognition 5, 73-99. 
Bulbulia, J. (2008). Meme infection or religious niche construction? an adaptationist alternative to the cultural maladaptationist hypothesis. Method and Theory in the Study of Religion 20, 1-42.

Butler, E. A. and Gross, J. J. (2009). Emotion and emotion regulation: integrating individual and social levels of analysis. Emotion Review 1, 86-87.

Caro Baroja, J. (1950). Una fiesta de San Juan en Castilla. Clavileño I 5, 57-64.

Christianson, S. A. (1992). Emotional stress and eyewitness memory: A critical review. Psychological Bulletin 12, 284-309.

Clarke, P. and Byrne, P. (1993). Religion defined and explained. Macmillan Press, London.

Collins, K. A., Pillemer, D. B., Ivcevic, Z., \& Gooze, R. A. (2007). Cultural scripts guide recall of intensely positive life events. Memory and Cognition 35, 651-659.

Cortés, L. (1969). Las fiestas de San Juan en San Pedro Manrique (Soria). Zephyrus 12, 171-185.

Crano, W. D. and Brewer, M. B. (2002). Principles and methods of social research. Lawrence Erlbaum Associates, Mahwah, NJ.

Ellis, H. C. and Ashbrook, P. W. (1989). The "state" of mood and memory research: A selective review. Journal of Social Behavior and Personality 4, 1-21.

Engle, R. W., Conway, A. R. A., Tuholski, S. W., \& Shisler, R. J. (1995). A resource account of inhibition. Psychological Science 6, 122-125.

Freyd, J. J. (1994). Betrayal trauma: Traumatic amnesia as an adaptive response to childhood abuse". Ethics and Behavior 4, 307-330.

Gross, J. J. (Ed.) (2007). Handbook of emotion regulation. Guilford, New York, NY.

Iñigues y Ortiz, M. (1924). Ritos celtibéricos. Las fiestas de San Pedro Manrique. Sociedad Española de Antropología, Etnografía y Prehistoria, Actas y Memorias III 23, 57-70.

Joseph, R. (1999). The neurology of traumatic "dissociative" amnesia: Commentary and literature review. Child Abuse and Neglect 23, 715-727.

Kikuchi, H., Fujii, T., Abe, N., Suzuki, M., Takagi, M., Mugikura, S., Takahashi, S., Mori, E. (2010). Memory repression: Brain mechanisms underlying dissociative amnesia. Journal of Cognitive Neuroscience 22, 602-613.

Kirchbaum, C., Wolff, O. T., May, M., Wippich, W. and Hellhammer, D. H. (1996). Stress- and treatment-induced elevations in cortisol levels associated with impaired declarative memory in healthy adults. Life Sciences 58, 1475-1483.

Konvalinka, I., Xygalatas, D., Bulbulia, J., Schjødt, U, Jegindø, E.-M., Wallot, S., Van Orden, G. and Roepstorff, A. (2011). Synchronized arousal between performers and related spectators in a firewalking ritual. Proceedings of the National Academy of Sciences of the United States of America $108,8514-8519$.

Liénard, P and Boyer, P. (2006). Whence collective rituals? A cultural selection model of ritualized behavior. American Anthropologist 108, 814-827.

Loftus, E. F. and Kaufman L. (1992). Why do traumatic experiences sometimes produce good memory (flashbulbs) and sometimes no memory (repression)?. In Winograd, E. and Neisser, U. (Eds.), Affect and accuracy in recall: Studies of "flashbulb" memories, pp. 212-223. Cambridge University Press, New York, NY.

Londeree, B. R. and Moeschberger, M. L. (1982). Effect of age and other factors on HR max. Research Quarterly for Exercise and Sport 53, 297-304.

Macrae, C. N., Bodanhausen, G. V. and Milne, A. B. (1998). Saying no to unwanted thoughts: Self-focus and the regulation of mental life. Journal of Personality and Social Psychology 74, 578-589.

McCauley, R. and Lawson, E. (2002). Bringing ritual to mind: Psychological foundations of religious forms. Cambridge University Press, Cambridge.

McClelland, J. (1997). Constructive memory and memory distortions: A parallel-distributed processing approach. In Schacter, D. L. (Ed.), Memory distortion: How minds, brains and societies reconstruct the past, pp. 69-9o. Harvard University Press, Cambridge, MA. 
Rappaport, R. (1999). Ritual and Religion in the Making of Humanity. Cambridge University Press, New York, NY.

Richards, J. M. (2004). The cognitive consequences of concealing feelings. Current Directions in Psychological Science 13, 131-134.

— and Gross, J. J. (1999). Composure at any cost? The cognitive consequences of emotion suppression. Personality and Social Psychology Bulletin 25, 1033-1044.

- (2000). Emotion regulation and memory: the cognitive costs of keeping one's cool. Journal of Personality and Social Psychology 79, 410-424.

- Butler, E. A. and Gross, J. J. (2003). Emotion regulation in romantic relationships: The cognitive consequences of concealing feelings. Journal of Social and Personal Relationships 20, 599-620.

Rimé, B. (2007). Interpersonal emotion regulation. In Gross, J. J. (Ed.), Handbook of emotion regulation, pp. 466-485. Guilford, New York, NY.

Robergs, R and Landwehr, R (2002). The surprising history of the "HRmax= 220-age" equation. Journal of Exercise Physiology Online 5 (2), 1-10.

Ruiz Vega, A. (1985). La Soria mágica. Fiestas y tradiciones populares. Ingrabel, Almazan, Soria.

San Baldomero Ucar, J. M. (1998). La fiesta de San Juan en San Pedro Manrique: Ensayo hermenéutico. Ayuntamiento de San Pedro Manrique, Soria.

Schjoedt, U., Stødkilde-Jørgensen, H., Geertz, A. and Roepstorff, A. (2011). The power of charisma: perceived charisma inhibits the attentional and executive systems of believers in intercessory prayer. Social Cognitive and Affective Neuroscience 4, 199-207.

— , Sørensen, J., Nielbo, K. L., Xygalatas, D., Mitkidis, P., Bulbulia, J. (2013). Cognitive resource depletion in religious interactions. Religion, Brain and Behavior 3, 39-55.

Talarico, J. M. and Rubin, D. R. (2003). Confidence, not consistency, characterizes flashbulb memories. Psychological Science 14, 455-461.

Vogeley, K. and Roepstorff, A. (2009). Contextualising culture and social cognition. Trends in Cognitive Science 13, 511-516.

Ward, C. (1979). Therapeutic aspects of ritual trance: The Shango cult in Trinidad. Journal for Altered States of Consciousness 5, 19-29.

Wegner, D. M., Erber, R. and Zanakos, S. (1993). Ironic processes in the mental control of mood and mood related thought. Journal of Personality and Social Psychology 58, 409-418.

Whitehouse, H. (1992). "Memorable religions: Transmission, codification and change in divergent melanesian contexts”. Man, New Series 27, 777-797.

- (2004). Modes of religiosity: A cognitive theory of religious transmission. AltaMira Press, Walnut Creek, CA.

Xygalatas, D. (2012). The burning saints: Cognition and culture in the fire-walking rituals of the Anastenaria. Equinox, London.

—, Konvalinka, I., Roepstorff, A. and Bulbulia, J. (2011). Quantifying collective effervescence: Heart-rate dynamics at a fire-walking ritual. Communicative \& Integrative Biology 4, 735-738.

Yerkes, R. M. and Dodson, J. (1908). The relation of strength of stimulus to rapidity of habitformation. Journal of Comparative Neurology and Psychology 18, 459-482. 